\title{
ANÁLISIS DEL ESTADO NUTRICIONAL EN ESTUDIANTES EDUCACIÓN FÍSICA, ASOCIADO A HÁBITOS ALIMENTARIOS Y NIVEL DE ACTIVIDAD FÍSICA.
}

\author{
THE NUTRITIONAL STATUS ANALYSIS IN PHYSICAL EDUCATION STUDENTS, ASSOCIATED WITH \\ EATING HABITS AND LEVEL OF PHYSICAL ACTIVITY.
}

\author{
Luis Daniel Antoniazzi', Laura Rosana Aballay²; Alicia Ruth Fernandez ${ }^{3}$, Marta Fiol de Cuneo 4.
}

\section{Resumen:}

Introducción: Numerosas investigaciones señalan que el estudiante universitario modifica sus hábitos alimentarios y su nivel de actividad física y, como consecuencia, su Índice de masa corporal (IMC) supera los valores saludables $(\geq 25)$. Objetivo general: Analizar el estado nutricional en los estudiantes de dos Profesorados de Educación Física de Córdoba, Argentina y su posible asociación con los hábitos alimentarios y el nivel de actividad física. Método: Estudio observacional, descriptivo y de corte transversal. Se estudiaron 134 estudiantes. Para valorar el estado nutricional se midió el IMC. Para indagar sobre la selección y las conductas alimentarias, se utilizó una encuesta basada en las recomendaciones de las guías alimentarias para la población argentina. Para estimar el gasto energético en actividad física se usó el Cuestionario Internacional de Actividad Física IPAQ, formato corto, versión en español. Resultados: El estado nutricional de la población estudiada mostró en general una mayor prevalencia con peso saludable (normopeso). Respecto a los hábitos alimentarios, las mayores proporciones de estudiantes evaluados presentaron una selección medianamente saludable y unas conductas saludables. En referencia al nivel de actividad física, ambos géneros mostraron, en una mayor proporción, un alto nivel de actividad física. Para todos los casos, el IMC resultó dentro de los valores saludables. Conclusiones: Se podría presumir que el saludable estado nutricional de la población estudiada se debe a sus hábitos alimentarios mayormente saludables y, principalmente, a su alto nivel de actividad física. No obstante, el seguimiento de esta población, en un estudio longitudinal, podría ratificar o rectificar estas primeras presunciones.

Palabras clave: estado nutricional; sobrepeso; estudiantes; educación física; hábitos alimentarios; actividad física.

\section{Abstract:}

Introduction: numerous studies show that the college-aged student modifies their eating habits and levels of physical activity and, consequently, their Body Mass Index (BMI) exceeds healthy values ( $\geq 25)$. General objective: to analyze the nutritional status in students of two Physical Education Teaching training Institutes, located in Cordoba, Argentina, and their possible association with eating behaviours and level of physical activity. Method: observational, cross-sectional and descriptive study. 134 students were studied.To assess nutritional status, the MBI was measured. To investigate about food selection and habits which accompanied the ingestion, a survey was used based on the recommendations from the Food Guidelines for the Argentine Population. To estimate energy expenditure by physical activity, the International Physical Activity Questionnaire (IPAQ) was used, in short format, in the Spanish version. Results: the nutritional status of the study population showed, in general, a higher prevalence with healthy weight (normal weight). As regards eating habits, for food selection as well as for eating habits, greater proportions of evaluated students showed a moderately healthy and healthy selection, respectively. In reference to the level of physical activity, both genres showed, in a larger proportion, a high level of physical activity. For both cases, the IMC resulted within healthy values. Conclusion: it can be assumed that the healthy nutritional status of the study population is due to their mostly healthy eating habits, and mainly to their high level of physical activity.

Keywords: nutritional status; overweight; students; physical education; eating habits; physical activity.

\footnotetext{
1 Licenciado en Fisioterapia y Kinesiología. Facultad de Educación Física. Universidad Provincial de Córdoba.

Email de contacto: antoniazziluis@hotmail.com

2 Doctora en Ciencias de la Salud. Escuela de Nutrición. Facultad de Ciencias Médicas. Universidad Nacional de Córdoba.

Email de contacto: laballay@fcm.unc.edu.ar

3 Doctorada en Medicina. Escuela de Salud Pública. Facultad de Ciencias Médicas. Universidad Nacional de Córdoba.

Email de contacto: aruthfernandez@gmail.com

4 Doctorada en Medicina y Cirugía. Facultad de Ciencias Médicas. Universidad Nacional de Córdoba.

Email de contacto: mhcuneo@hotmail.com
} 


\section{Introducción}

El sobrepeso y la obesidad se manifiestan en edades cada vez más precoces. Según un estudio publicado en los Archivos Argentinos de Pediatría, ya en el año 2003, el 26,2\% de niños y adolescentes superaban ampliamente el índice de masa corporal estimado para su sexo y edad ${ }^{1}$. Al respecto, factores determinantes como el nivel socioeconómico alto, la residencia en las ciudades metropolitanas, el género femenino, el desconocimiento y las falsas creencias acerca de la nutrición, el marketing de empresas transnacionales de alimentos, el creciente estrés académico y las pobres instalaciones para actividades físicas han conducido al aumento de la obesidad infantil en los países en desarrollo, por caso Argentina en un $19,3 \%{ }^{2}$. Todos estos factores magnifican los problemas resultantes de la transición epidemiológica y hacen que la edad en que aparecen las enfermedades crónicas, asociadas con la alimentación, sea cada vez más temprana y en grupos de menores ingresos ${ }^{3}$. Un estudio poblacional en Argentina entre 20052006, con una muestra de 4567 personas, concluyó que el sobrepeso alcanzaba el 52\%; en tanto que la cantidad de personas obesas llegaba al $17 \%{ }^{4}$. En una revisión actualizada que relaciona el síndrome metabólico, la enfermedad cardiovascular y el cáncer en América del Sur, se establece que la obesidad y el cáncer pueden ser evitables, al menos en parte, por el comportamiento saludable. Plantea además que el ejercicio, el control de peso y los hábitos alimentarios saludables son importantes para reducir el riesgo de las principales enfermedades crónicas. La citada revisión concluye que, para prevenir las enfermedades relacionadas con la dieta y el estilo de vida en América del Sur, es imprescindible incluir educación nutricional y hábitos de vida saludables ${ }^{5}$. Según proyecciones más actuales de la Sociedad Argentina de Cardiología, la obesidad está creciendo más aceleradamente en América Latina que en los países desarrollados. En nuestro país, no existen hasta el momento estudios que permitan proyectar la situación en el futuro. En un modelo de simulación, se estimó que la prevalencia de obesidad en adultos para 2027 en la Argentina será del $25,9 \%{ }^{6}$. Un estudio más específico sobre estudiantes de la Licenciatura en Educación Física, de la ciudad de Valparaíso, Chile, determinó que el ejercicio físico incide sobre la estructura corporal, así como la morfoestructura influye sobre el rendimiento. La estructura corporal del estudiante universitario de Educación Física cobra importancia, entonces, debido a que será relevante en el logro de asignaturas que impliquen rendimiento físico ${ }^{7}$. Por su parte, otro estudio confirma que la elevada proporción de grasa abdominal es un factor de riesgo mayor para el desarrollo de la enfermedad coronaria, la diabetes tipo II y la mortalidad relacionada con esto ${ }^{8}$.

Con respecto a la importancia de la actividad física, el papel del sedentarismo como factor de riesgo cardiovascular ha sido muy estudiado en las últimas cuatro décadas. Los resultados de estos trabajos muestran un descenso de la prevalencia de enfermedades crónicas como diabetes, obesidad, enfermedad cardiovascular, osteoporosis, síndrome metabólico e, incluso, algunas neoplasias en aquellos sujetos físicamente activos $^{9-11}$. Específicamente en estudiantes de carreras no relacionadas con la salud, en el plano internacional, un estudio comparativo, durante los años 1999 y 2001, involucró a 23 países de Sudamérica con diferentes características. Este estudio relevó información acerca de la frecuencia de la actividad física realizada en el tiempo libre. Se encontró que la prevalencia de insuficiente actividad física varió de acuerdo a las condiciones culturales y económicas de cada país, siendo del 23 \% para Europa Noroccidental y del $44 \%$ para países en desarrollo como Venezuela, Colombia y Sudáfrica ${ }^{12}$. Utilizando el mismo indicador, en un estudio similar realizado en Argentina, resultó una prevalencia del $36 \%$ en actividad física nula durante el tiempo libre. En la misma investigación, ahora con indicadores propios, se encontró que un nivel de actividad física alto sólo es alcanzado por el $40 \%$ de los estudiantes ${ }^{13}$. En lo que refiere a estudiantes de carreras afines a la salud, particularmente a la Educación Física, el citado estudio menciona trabajos realizados en institutos públicos de formación docente de Buenos Aires. Éstos fueron desarrollados en el ISEF N $N^{\circ}$ "E. R. Brest" y en el ISEF N 2 "F. W. Dickens" de dicha ciudad. Entre sus resultados, se observó que el $88 \%$ de los estudiantes sondeados reportaron un nivel alto de actividad física habitual y, además, que el nivel de actividad física de los estudiantes de Educación Física del profesorado privado de la Universidad de Flores resultó ser similar al de los estudiantes de profesorados públicos ${ }^{14,15}$.

De acuerdo a los antecedentes expuestos, el sobrepeso y la obesidad, como pandemia que ocasiona complicaciones en la salud, se encuentran presentes en muchas poblaciones de estudiantes de edad universitaria, asociados a patrones alimentarios e inactividad física. En virtud de esto, el objetivo de este 
trabajo fue analizar el estado nutricional de los estudiantes de Profesorados de Educación Física y su posible asociación a las conductas alimentarias y la actividad física, en los Institutos de formación IPEF y San Miguel, ambas instituciones con sede en la Ciudad de Córdoba, Argentina. El estudio de estos hábitos en los futuros profesores de Educación Física permitiría acreditar objetivamente aspectos referidos al estilo de vida en individuos con una potencial influencia en la prevención de la obesidad en niños y adolescentes.

\section{Método}

Estudio observacional, descriptivo y de corte transversal. Se llevó a cabo en el Instituto Provincial de Educación Física (IPEF) y en el Instituto Privado San Miguel (ISM), ambas instituciones de la Ciudad de Córdoba, Argentina. Se evaluó durante el período abril a junio de 2015. La selección de la muestra se hizo de manera aleatoria simple sobre una población de $n=633$, estimando una muestra de 134 estudiantes de los institutos mencionados. Fueron excluidos de este estudio los estudiantes que se encontraban realizando una dieta alimentaria restrictiva por razones de salud; los estudiantes que se encontraban en recuperación física por alguna lesión o enfermedad y las estudiantes con embarazos de más de diez semanas. En todos los casos, estos factores se consideraron posibles modificadores del Índice de masa corporal (IMC). Las variables principales fueron los hábitos alimentarios y el nivel de actividad física; y como variable dependiente el IMC. Además, se consideró como variable de ajuste, el sexo. Como instrumentos de evaluación antropométrica, se utilizaron una cinta de acero flexible, ancho de $7 \mathrm{~mm} .{ }^{16} \mathrm{y}$ una balanza de contrapesos con capacidad para $150 \mathrm{~kg}$ y con una precisión cada 100 gs, con estadiómetro incluido. Para estimar el gasto energético en actividad física se usó el Cuestionario Internacional de Actividad Física, formato corto ${ }^{17}$. Para la valoración de los hábitos alimentarios se construyó una encuesta basada en las recomendaciones de la actual guía alimentaria para la población argentina ${ }^{18}$. Esta constaba de dos escalas de valoración; en una se consideró la selección de alimentos y su frecuencia de consumo semanal. En la otra, se consideraron las conductas alimentarias que acompañaron las ingestas. Según la totalidad de las respuestas, se categorizaron los hábitos alimentarios en: saludable, medianamente saludable y no saludable. En la escala de valoración de selección y frecuencia de consumo de alimentos, se los dividieron a los alimentos en tres grupos, en función de su consumo recomendado. En el primer grupo, se encontraban los alimentos que se consumen más de tres veces por semana; en el segundo grupo, los alimentos que se consumen hasta tres veces por semana y, en el tercer grupo, los que se recomienda no consumir. A modo de ejemplo se consideró que, cuando se consumen alimentos del primer y segundo grupo, se le asigna el valor 1 y en caso contrario el valor 0 . Para el tercer grupo, se utilizó un criterio en orden inverso de puntuación; es decir, se asignaba el valor 1, cuando se no consumían los alimentos de este grupo. De este modo quedó constituida una escala con un rango de 0 a 7 puntos; en la cual, una puntuación $>5$ se consideró como "saludable"; una puntuación entre 3 y 4 se consideró "medianamente saludable" y una puntuación $<3$ se consideró "no saludable". En la escala que valoraba las conductas alimentarias, a éstas se las dividieron en siete puntos, en función de las recomendaciones saludables y se les otorgó la valoración de 1 por cada conducta seleccionada. Al igual que la anterior escala, quedó constituida con un rango de 0 a 7 puntos. En este caso, una puntuación $>6$ se consideró como "saludable"; una puntuación entre 3 y 5 se consideró "medianamente saludable" y una puntuación < 3 se consideró "no saludable". Las variables fueron expresadas como media y desviación estándar. Para comparación de medias se utilizo ANOVA. Para la asociación de variables categóricas se utilizó test de chi cuadrado y se aplico test de Fisher cuando fue necesario. Un valor de $p<0,05$ fue considerado significativo. El análisis estadístico fue realizado utilizando Infostat versión 2016, UNC. Los datos se recolectaron de manera grupal en un aula sin control de tiempo, con la autorización de cada estudiante mediante una carta de consentimiento informado. El presente trabajo fue autorizado por el Comité Internacional de Ética de las Investigaciones en Salud (CIEIS), del Hospital Nacional del Clínicas, de la Universidad Nacional de Córdoba; y no cuenta con aportes económicos ni subsidios de ningún tipo. No obstante, las Sras. Mónica Garro y María Eugenia Gallastegui, directoras de las instituciones donde se realizó este estudio, ofrecieron la plena disponibilidad de las instalaciones de ambos establecimientos educativos para la recolección de los datos. Asimismo, la Dra. Marta Fiol, directora del presente trabajo, aportará la infraestructura necesaria y el acceso informático como recurso técnico para la prosecución de este trabajo. 


\section{Resultados}

El estado nutricional de la población estudiada mostró en general una mayor prevalencia con peso saludable (normopeso). Respecto a la comparación por sexos, las mujeres presentaron similar prevalencia de normopeso que los varones. (Tabla 1)

Tabla 1: Índice de masa corporal (IMC), normopeso y sobrepeso, en muestra total y según sexo, en estudiantes del Instituto Provincial de Educación Física (IPEF) y del Instituto San Miguel (Córdoba, Argentina).

\begin{tabular}{l|c|c|c|c}
\hline Muestra & FA & $\begin{array}{c}\text { IMC total } \\
\text { Media } \pm \text { DE }\end{array}$ & $\begin{array}{c}\text { Normopeso } \\
\text { (IMC<24,99) } \\
\text { FA (\%) }\end{array}$ & $\begin{array}{c}\text { Sobrepeso (IMC>25) } \\
\text { FA (\%) }\end{array}$ \\
\hline Ambos sexos & 134 & $23,35 \pm 3,08$ & $104(78)$ & $30(22)$ \\
Mujeres & 60 & $23,08 \pm 3,03$ & $48(80)$ & $12(20)$ \\
Varones & 74 & $23,57 \pm 3,13$ & $56(76)$ & $18(34)$ \\
\hline \multicolumn{2}{c}{ Las variables se expresan como Media \pm Desviación estándar (DE), FA: frecuencia absoluta, entre paréntesis, porcentaje de casos. }
\end{tabular}

Respecto a los hábitos alimentarios, en particular para la selección de alimentos, las mayores proporciones se corresponden a una selección medianamente saludable $(p=0,0001)$, con un IMC dentro de los valores saludables. (Tabla 2)

En relación a la conducta alimentaria, la mayoría de los evaluados, en este caso presentó una conducta saludable $(p=0,0001)$. También aquí el IMC se encuentra dentro de valores saludables. (Tabla 2)

En referencia al nivel de actividad física, ambos géneros muestran, en una mayor proporción, un alto nivel de actividad física $(p=0,0001)$ y, al igual que en los casos anteriores, sus valores de IMC son saludables. (Tabla 2)

Tabla 2: Distribución de hábitos alimentarios y nivel de actividad física con Índice de masa corporal (IMC) en estudiantes del Instituto Provincial de Educación Física (IPEF) y del

Instituto San Miguel (Córdoba, Argentina).

\begin{tabular}{|c|c|c|c|}
\hline & Variables & $\mathrm{FA}(\%)$ & $\mathrm{IMC}($ Media $\pm \mathrm{DE})$ \\
\hline $\begin{array}{l}\text { Selección } \\
\text { alimentaria }\end{array}$ & $\begin{array}{l}\text { Saludable } \\
\text { Medianamente saludable } \\
\text { No saludable }\end{array}$ & $\begin{array}{ll}30 & (22) \\
94 & (70) \\
10 & (8)\end{array}$ & $\begin{array}{l}22,47 \pm 2,51 \\
23,64 \pm 3,11 \\
23,25 \pm 4,07\end{array}$ \\
\hline $\begin{array}{l}\text { Conducta } \\
\text { alimentaria }\end{array}$ & $\begin{array}{l}\text { Saludable } \\
\text { Medianamente saludable } \\
\text { No saludable }\end{array}$ & $\begin{array}{cc}87 & (65) \\
43 & (32) \\
4 & (3)\end{array}$ & $\begin{array}{l}23,09 \pm 2,79 \\
23,67 \pm 3,54 \\
25,49 \pm 3,69\end{array}$ \\
\hline $\begin{array}{l}\text { Nivel de } \\
\text { Actividad } \\
\text { Física }\end{array}$ & $\begin{array}{l}\text { Alto } \\
\text { Medio } \\
\text { Bajo }\end{array}$ & $\begin{array}{cc}97 & (72) \\
26 & (20) \\
11 & (8)\end{array}$ & $\begin{array}{l}23,37 \pm 2,83 \\
22,78 \pm 3,15 \\
24,52 \pm 4,72\end{array}$ \\
\hline
\end{tabular}

\section{Discusión}

Conforme con los resultados, se detectó que la población estudiada, presentó un porcentaje de normopeso por encima de los valores representativos de la población argentina ${ }^{19,20}$. En este sentido, es para tener en cuenta que las mediciones de los evaluados se corresponden al inicio del cursado de la carrera, cuando podrían aún no haber incorporado los hábitos alimentarios de un estudiante más avanzado. Por otra parte, la selección de alimentos resultó ser mayoritariamente medianamente saludable, consumiendo en algunas ocasiones alimentos ultraprocesados o productos de pastelería y, en otros casos, careciendo de macronutrientes esenciales como carnes, huevos, vegetales, frutas y semillas. Es importante destacar que en las sedes educativas, donde cursan los estudiantes evaluados, los comedores disponibles, no presentan alternativas más o menos saludables; lo que haría suponer que el patrón de selección alimentaria resulta igual que fuera de los citados establecimientos. Por su parte, las conductas alimentarias 
fueron mayormente saludables, respetando 4 ingestas diarias mínimas, asegurando el desayuno y el almuerzo, y evitando los ayunos prolongados, como las más destacadas. Este resultado no fue coincidente con los hallazgos obtenidos en estudiantes universitarios de Colombia y España, referidos a los cambios en el estilo de vida, reflejados en los hábitos alimentarios y en la calidad de la dieta, respectivamente ${ }^{21,22}$. En el mismo sentido, en la Ciudad de Córdoba, Argentina, un estudio reconoció que una ingesta diaria superior o inferior a lo recomendado y el bajo nivel de actividad física, como variables relacionadas con el sobrepeso ${ }^{23}$. Respecto a esta discrepancia con los resultados encontrados en el presente estudio, cabe agregar que la currícula de la carrera de Educación Física no presenta contenidos específicos relacionados a hábitos alimentarios saludables; lo que haría presumir que las conductas manifestadas son adquiridas de manera espontánea desde la infancia y la adolescencia. En relación al nivel de actividad física, la mayoría de los estudiantes evaluados mostraron un alto nivel de actividad física. Posiblemente en este caso, los contenidos estrictos relacionados con el movimiento humano, que presenta la formación académica de Educación Física, sean la causa del alto nivel de actividad física observado en la población analizada. Por la misma presunción, podría verse el contraste del marcado sedentarismo, en el estilo de vida de estudiantes universitarios de latinoamérica ${ }^{24}$.

\section{Conclusión}

Los estudiantes evaluados presentaron una mayor prevalencia de normopeso, con valores que mostraron asociación con hábitos alimentarios mayormente saludables y con altos niveles de actividad física. A los fines de confirmar estas primeras afirmaciones, sería oportuno y pertinente replicar este estudio, considerando otras variables, como el contexto sociocultural, la historia motriz y los antecedentes genéticos. Asimismo, resultaría valioso continuar estudiando a esta población durante el cursado de la carrera, para ratificar o rectificar las actuales presunciones.

\section{Bibliografía}

1. Kovalskys I, Bay L, Rausch Herscovici CB. Prevalencia de obesidad en una población de 10 a 19 años en la consulta pediátrica. Arch Argent Pediat. 2003; 101(6): 441-47.

2. Gupta N, Goel K, Shah P, Misra A. Childhood obesity in developing countries: epidemiology, determinants, and prevention. Endocr Rev. 2012; 33(1): 48-70.

3. Lang T, Ducimetiere P. Premature Cardiovascular Mortality in France. Int J Epidemiol 1995; 24(2): $331-339$.

4. Aballay LR, Osella AR, Celi $A$, et al. Overweigth and obesity: prevalence and their association with some social characteristics in a random sample population based study in Cordoba City, Argentina. Obes Res Clin Pract. 2009; 3, 75-83

5. Aballay LR, Eynard AR, Díaz Mdel P, Navarro A, Muñoz SE. Overweight and obesity: a review of their relationship to metabolic syndrome, cardiovascular disease, and cancer in South America. Nutrition Reviews. 2013;71(3):168-79.

6. Cardone A, Borracci RA, Milin E. Estimación a largo plazo de la prevalencia de la obesidad en la Argentina. Rev Argent Cardiol 2010; 78(1): 23-29.

7. Almagià Flores, AA, Lizana Arce PJ, Rodríguez Rodríguez FJ, Marincovich DI, Binvignat Gutiérrez O. Variables Antropométricas y Rendimiento Físico en Estudiantes Universitarios de Educación Física. Int J Morphol 2009; $27(4): 971-975$.

8. Kissebah AH, Freedman DS, Pieris AN. Health risks of obesity. Medical Clinics of North America. 1989; 73(1): $111-138$.

9. William B. Kannel, MD; Paul Sorlie, MS. Some health benefits of physical activity. The Framingham Study. Arch Intern Med. 1979; 139(8): 857- 861.

10. Wingard $D$. The sex differential in mortality rates: demographic and behavioral factors. Am J Epidemiol. 1982; 115(2): 205216.

11. Rennie KL, McCarthy N, Yazdgerdi S, Marmot M, Brunner E. Association of the metabolic syndrome with both vigorous and moderate physical activity. Int J Epidemiol. 2003; 32(4): 600-606.

12. Haase A, Steptoe A, Sallis JF, Wardle J. Leisure-time physical activity in university students from 23 countries: associations with health beliefs, risk awareness, and national economic development. Prev Med. 2004; 39(1): 182-190.

13. Farinola MG, Bazán NE. Sedentary behavior and physical activity in university students:a pilot study. . Rev Argent Cardiol. 2011; 79(4): 351-354.

14. Farinola M. Nivel de actividad física en estudiantes universitarios con especial referencia a estudiantes de profesorado en educación física. Revista electrónica de Ciencias Aplicadas al Deporte. 2011; 4(12): 1-12.

15. Farinola M, Polo M, La Valle L. Niveles de actividad física en alumnos/as del Instituto de Educacion Física NNo 2 Federico W. DICKENS. Revista Electrónica de Ciencias Aplicadas al Deporte. 2009; 2(5): 1-9.

16. Seidell J, Kahn H, Williamson D, Lissner L, Valdez R. Report from a Centers for Disease Control and Prevention Workshop on Use of Adult Anthropometry for Public Health and Primary Health Care. Am J Clin Nutr. 2001; 73(1): $123-6$.

17. IPAQ. International Physical Activity Questionnaire. 2005. Disponible en https://sites.google.com/site/theipaq/home

18. Guías Alimentarias para la Población Argentina. Disponible http://www.msal.gob.ar/images/stories/bes/graficos/0000000817cnt-2016-04 Guia Alimentaria completa web.pdf

19. Tercera Encuesta Nacional de Factores de Riesgo. Disponible en http://www.msal.gob.ar/images/stories/bes/graficos/0000000544cnt-2015_09_04_encuesta_nacional_factores_riesgo.pdf

20. Guía práctica clinica nacional sobre diagnostico y tratamiento de la obesidad en adultos. Disponible en http://www.msal.gob.ar/images/stories/bes/graficos/0000000971cnt-2017-06_guia-practica-clinica_obesidad.pdf 


\section{ESTADO NUTRICIONAL EN ESTUDIANTES.}

21. Edgar Ibáñez, Yeily Thomas, Angela Bicenty, Judith Barrera, Jeannette Martínez, Ruby Gerena. Cambios de hábitos alimentarios de los estudiantes de odontología de la Fundación universitaria San Martin de Bogotá, Colombia. Disponible en http://www.unicolmayor.edu.co/publicaciones/index.php/nova/article/view/103

22. Arroyo Izaga M, Rocandio P, Ansotegui Alday L, Pascual Apalauza E, Salces Beti I, Rebato Ochoa E. Calidad de la dieta, sobrepeso y obesidad en estudiantes universitarios. Nutrición Hospitalaria. 2006: 19(6)

23. Pi Romina, Vidal Paula, Brassesco Bárbara, Viola Lorena, Aballay Laura. Estado nutricional en estudiantes universitarios: su relación con el número de ingestas alimentarias diarias y el consumo de macronutrientes. Nutrición hospitalaria. 2015: 31(4) 1748-1756.

24. Rangel Caballero, Luis Gabriel, Gamboa Delgado, Edna Magaly, Murillo López, Alba Liliana, Prevalencia de factores de riesgo comportamentales modificables asociados a enfermedades no transmisibles en estudiantes universitarios latinoamericanos: una revisión sistemática. Nutrición Hospitalaria [en linea] 2017, 34. 